\title{
A Comparative Study on Mathematical Modeling of Consciousness within Creation
}

\author{
Somyajit Chakraborty ${ }^{\mathrm{a}}$ \\ ${ }^{a}$ Department of Computer Science and Engineering, Techno India University, West Bengal
}

\begin{abstract}
In this paper we shall review the approaches to mathematically formulate cognitive thought processes or consciousness made by Yinsheng(2008) and Bhushan(2018). In the first section of this paper, we will provide a brief insight on both the discrete approach to general consciousness made by Yinsheng and the quantum mechanical approach towards information consciousness made by Bhushan. In the following section, we will thoroughly discuss the implication of the above theories as well as some other theories like Orch-OR theory of consciousness by Hameroff and Penrose(2013)

Keywords: consciousness, information consciousness, discrete group theory, line integral loop dynamics, orchestrated objective reduction, mathematical interpretation, Review paper
\end{abstract}

\section{Introduction}

The term 'consciousness', in its very foundation, has deep philosophical as well as scientific meaning. Consciousness, though complex in nature, yet it is something which all life-form possesses in one form or the other. Over time, several attempts have been made to define consciousness through different subjects. Thus, in order to avoid a complex-endless debate on defining consciousness we shall discuss the regulations that were proposed to define consciousness.

\footnotetext{
${ }^{\star}$ Review on Mathematical Models of Consciousness

Email address: c, orcid=0000-0002-2038-5169 (Somyajit Chakraborty)
} 
Definition 1: Consciousness is not an independent quality but arose, in terms of conventional physical processes, as a natural evolutionary consequence of the biological adaptation of brains and nervous systems. 4.

Definition 2: Definition of consciousness must be acceptable both for philosophy and science. The person defining, should be clear that the definition of the word "consciousness" is just one as both the philosophers and the scientists refer to, there should not be the separate interpretation of the word "consciousness" from other study fields. [1]

Definition 3: The concept of consciousness must be common for any type of the matter to be tested by the definition-standard, in other word, no matter human, animal or machine it is, if it satisfies the definition-standard of consciousness, it should be regarded as being conscious. [1]

With these ideas in mind we should be able to understand the common causality or nature of consciousness. Norman Malcolm, in his study, explained the common meaning behind the above ideas. He pointed out that "being conscious" can be understood in two different ways. The first understanding is called transitive, because this form of consciousness takes an object, which means, it is conscious of something. The second use is called intransitive, because this form of consciousness has no object 3 .

In summary, we have three main understanding addressed by complex cognitive thought process or consciousness, they are:

- Consciousness in the realm of creation, is a consequence of biological adaptation.

- Consciousness in the realm of spirituality, is a distinct physical quality which exists in perpetuity.

- Consciousness in the realm of science, is an essential ingredient of physical laws not yet fully understood. 


\section{Information Consciousness:}

The idea behind information consciousness has been explored more recently. Consciousness as an information process over time and space can very well be defined as, Access Consciousness, which is the time extended form of processes in phenomenal consciousness and self-consciousness to the time extended form of physical processes. In fact, Decartes, Lockem, Hume etc. described this process as if there is an observer in their mind looking out at qualia of feeling qualia in space and time. Most descriptions of consciousness or cognitive thought process include continuity for time and representation for space. The empirical evidence of Kant and Descartes are the bedrocks of consciousness studies where it is explained as an observation containing space, time and mind. Kant (1781) had further argued that our minds must be capable of representing objects in space and time where experiences presuppose space and time as pure concepts of reason. Without space, objects could not be differentiated and would have no properties. Similarly, without representation in time, the concepts of succession of events and simultaneity would be unknown to us. In this way, the mind may also have physical intuition of the meaning of its space, time and content so that the qualia become grouped in objects, events, memory, realization including imaginings and perceptions. Giulio Tononi's Integrated Information Theory (IIT) [6] also offers an explanation for the nature and source of consciousness being identical to a certain kind of information expressible in the form of binary digits $(0,1)$, the realisation of which requires physical, not merely functional, integration, and which can be measured mathematically according to the phi metric. As an equivalence, John Archibald Wheeler had also characterized the idea as "It from bit" — "it" referring to all the stuff of the universe and "bit" meaning information 9 .

\section{Homomorphic Model of Consciousness}

In this section we shall discuss the homomorphic model of consciousness proposed by Yinsheng, in 2008 using group theory [1]. The clear declaration 
that consciousness can be modeled as homomorphism of a group was made by Jean Piaget which proved the thought of Norman Malcolm about transitivity of consciousness.

The model presented by professor Yinsheng explored how homomorphic group theory can be used for interpreting Consciousness or Cognitive Thought Process. He started by defining homomorphism of groups, which is widely accepted by the mathematical community:

Let $\mathrm{G}_{1}\left(\mathrm{X},{ }^{\circ}\right)$ and $\mathrm{G}_{2}\left(\mathrm{Y},{ }^{*}\right)$ be the two groups, $\mathrm{f}$ : $\mathrm{X} 221 \mathrm{Y}$ is a mapping, if for any element $a, b \in X$, there must be

$$
f(a \circ b)=f(a) * f(b)
$$

Then, $\mathrm{f}: \mathrm{X} 221 \mathrm{Y}$ is defined as homomorphism from $\left(\mathrm{X},{ }^{\circ}\right)$ to $\left(\mathrm{Y},{ }^{*}\right)$. He described if $\mathrm{G}_{1}\left(\mathrm{X},{ }^{\circ}\right)$ be an information system $\mathrm{F}, \mathrm{X}$ is information, ${ }^{\circ}$ be an operator of information, $\mathrm{G}_{2}\left(\mathrm{Y},{ }^{*}\right)$ is the system of entity, $\mathrm{Y}$ is the set of entities outside of the information such as the substance, ${ }^{*}$ is the type of movement or relation of the entities, there is a set $\mathrm{F}$, among $\mathrm{F}$, there is element $\mathrm{f}$ : $\mathrm{X} 221 \mathrm{Y}$ is a mapping, if there is the elements $\mathrm{x}_{1}, \mathrm{x}_{2}, \mathrm{x}_{3}, \ldots, \mathrm{x}_{n}$ belongs to $\mathrm{X}$ such that $\mathrm{n}>0$, so that:

$$
f\left(X_{1} \circ X_{2} \circ X_{3} \ldots X_{n}\right)=f\left(X_{1}\right) * f\left(X_{2}\right) * f\left(X_{3}\right) * \ldots f\left(X_{n}\right)
$$

Then we get the system $\mathrm{F}$ cognizing the set $\mathrm{F}$ as the consciousness system, i.e., we say $\mathrm{F}$ is conscious or $\mathrm{F}$ has consciousness.

\subsection{Discussion}

This vision of consciousness is truly a remarkable envision. But Consciousness in its essence has a deeper Quantum nature if we are considering the thought process in its entirety, as a result of which the above model will face several challenges both mathematically and philosophically. In the paper, there is an example: 'The cup is on the table" is described as $\mathrm{f}($ the, cup, the table, on, is ,$^{\circ}$ ) is conscious for that:

$f($ the cup is on the table $)=f($ the cup $) * f($ the table $) * f($ on $) * f(b e)$ 
Which is equivalent to the real circumstance that the cup is on the table. here ${ }^{\circ}$ signifies permutation of the words according to grammar, and * signifies space construction of entities of the cup and the table. This Cognitive Thought on the sentence "the cup is on the table" essentially involves the notion of recognition of the spatial arrangement of the cup(the object), this feature describes the entities of the cup. But in a perturbative universe, the idea of space is quite relative to the observer and can affect the computation of certain processes, mostly cognitive processes. If we decide that the sentence of our discussion is absolute then we can ignore perturbations on the spatial recognition of our object 'cup'. But like any other sentence if we agree that the sentence is just a notion the spatial perturbations will come into play meaning questions will arise like, "With respect to whom the cup is on the table?" , "Is the observer the same as the person who wrote or commented the sentence?" , etc. To explore such questions we need a quantum mechanical version of the above model. That is why we move forward to a quantum mechanical approach described by Bhushan in 2018 2.

\section{Field Interpretation of Information Consciousness}

We have already defined the scientific philosophy behind information consciousness in section-2 of our paper, as described by Tononi $[6$. In this section we shall now discuss the quantum mechanical approach provided by Bhushan in 2018 2]. In his paper, he explored a mathematical interpretation to express the dynamics about the origin and characteristics of information consciousness. His proposal is quite essential and important as it reflects all the important theories

on information consciousness like, Orch-Objective Reduction Theory (Hameroff and Penrose) [4, Integrated Information theory (Giulio Tononi) [7] and others.

Mathematically, Information consciousness can be described as a function of transformation of potential information consciousness into kinetic consciousness i.e. $\mathrm{f}\left(\mathrm{C}_{0}+\mathrm{C}_{1}\right)$ which can further be assumed to be a divergence operator explaining how much the consciousness field gradient tends to spread radially 
outward or converge inward; a kind of behavior observed under the Gauss ${ }^{6}$ law of electric field lines charging or spreading out from charges in every direction. In other way, the consciousness field can be fully characterized by its divergence and curl where Potential consciousness energy is termed to be self-absorbed $\left(\mathrm{C}_{0}\right)$ whereas Kinetic energy is a manifest form of information self-awareness as $\mathrm{C}_{1}$. We describe this single bit of quantum information (Qubit) using two real numbers involving infinitely many decimal points in between them.So, $\Delta \mathrm{C}^{0 \leftrightarrow 1}$ movement from zero to one also includes the fundamental property of infinitely big or small quantities as infinity $\infty / \infty=1$ and one can always add a subsequent ' 1 ' to any number or quantity to make it further bigger or larger. And, once we put $\Delta \mathrm{C}^{0 \leftrightarrow 1}$ over $(S, T)^{\infty}$ i.e Space-time, things start demonstrating divergence as $\frac{\triangle C^{0 \leftrightarrow 1}}{(S, T)^{\infty}}$

In graph theoretical terms, $(\mathrm{S}, \mathrm{T})$ is an ordered pair comprising a space-set $\mathrm{S}$ of vertices or nodes or points or regions representing Cosmos made up of infinite galaxies or multiverses bounded together with a time set $\mathrm{T}$ of edges or $\operatorname{arcs}$ or lines or motion/ movement between the vertices stretching to infinity. It can also be visualized as a Cosmic Topology. The proper representation why (S,T), space-time is infinite in nature is given in Figure 1

Therefore, 'C'(Information Consciousness) can be represented as an Integral expressing change of consciousness from 0 (Global source-Potential Energy) to 1 (Local sink-localisation of kinetic energy). Therefore, information consciousness can be perceived as being binary coded digits of information-bits of 0 and 1 and therefore we can now explain, the entire mechanism of creation or evolution resulting due to a continuous transition, change or shift of the information consciousness between 0 and 1 as denoted by $\Delta \mathrm{C}^{0 \leftrightarrow 1}$ or $\int_{0}^{1} C$

For an information object moving along a closed loop path $\mathrm{C}$ in a consciousness vector field $\mathrm{F}(\mathrm{x}, \mathrm{y})$, the total work done by the field on the object is obtained by summing up the differential work done in moving from one point to another through a function which return the outward unit normal vector at some point on the curve C. This gives the line integral concept of information 


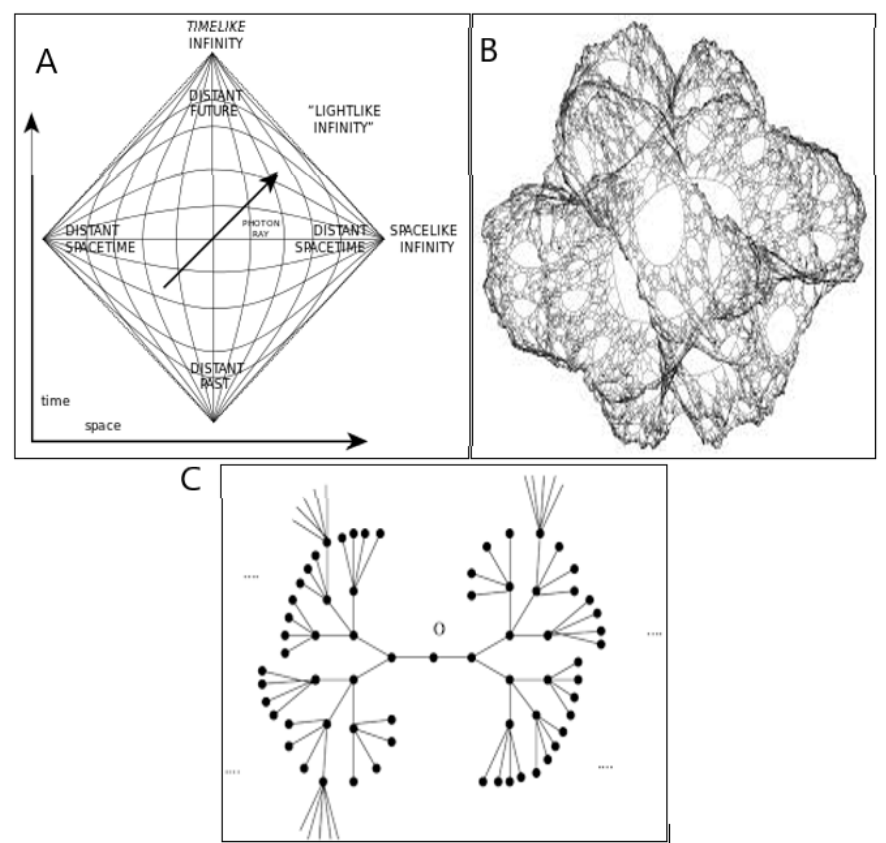

Figure 1: A. Penrose-diagram of space-time. B. Network of infinite series of Space-Time Extension. C. Graph with at least 2 edges going to infinity-Gauss-Bonnet operator of an infinite graph 10

consciousness and can be expressed as -

$$
\oint_{C} F \cdot d r
$$

Now like any conservation of field, theoretically the closed integral in equation 3 should now be equal to zero, since it is a closed loop. With this modification, let us now introduce the infinite nature of space(dr) and time(dt). So the equation we get should be an infinite series of information consciousness cycles spread over space-time:Clearly,

$$
\oint_{C \rightarrow \infty} F \cdot d r \cdot d t=0
$$

Clearly,

$$
\underset{C_{1}+C_{2}+\ldots+C_{n} \rightarrow \infty}{\oint} F \cdot d r \cdot d t=0
$$

Or,

$$
f(C, S, T)=\frac{\triangle C^{0 \leftrightarrow 1}}{(S, C)^{\infty}}
$$


Including the information consciousness cycle the equation modifies to:

$$
f(C, S, T)=\frac{\int_{0}^{1} C \cdot d C}{(S(C, T) T)^{\infty}}=\text { Diverges }
$$

The final equation conveys that creation consciousness is evolutionary over infinite space-time, and movement of information from 0 to 1 is a creation process and movement from 1 to 0 is destructive. "Qualia of consciousness" are those many moments of such experiences under the space-time fabric of holistic creation realized subjectively by more sentient entities than other lesser/ inanimate ones. (Penrose-Hameroff Orchestrated reduction theory) [4].

\section{Review Discussion}

In this section we shall discuss how both the theories above described is very much related, despite of different origination. Philosophically speaking consciousness has both discrete and quantum mechanical approach to it. The perturbations which could not be described by professor Yinsheng's approach, if combined with Bhushan's Field-Theoretical approach can be easily explained due to presence of mathematical geometry in Penrose-model $[8]$ as shown in figure-

1 of space-time in it. The interpretation that space-time $(\mathrm{S}, \mathrm{T})$ is an infinite graph is a key factor in describing consciousness, which to any observer, even the entity which is conscious, is continually infinite. If we can combine the discrete nature of consciousness with the evolutionary creation consciousness we might be able to quantify and explain the singularities, like Penrose-stairs [5], which originates due to quantum fluctuation, existing on the conscious field as explored by Bhushan. We can now, explain the discrete input process system, though parallel, of the signals or parameters affecting consciousness as explored by Yinsheng.

\section{Conclusion}

In this section I would like to conclude that information consciousness in its fundamental form, may it be evolutionary or discrete or destructive, yet exists, 
can therefore be modeled in a unified theoretical manner if we combine the discrete homomorphic model of consciousness along with the quantum mechanical field interpretation of information consciousness discussed in this paper.

\section{Acknowledgments}

I would like to acknowledge the assistance of my mentor Avijit Gayen, of Department of Computer Science and Engineering for great insight on the topic and I would also like to acknowledge the support of professor Shang Yinshen of Institute of Scientific and Technical Information of China, who has been a constant help in putting together this review paper.

\section{References}

[1] Zhang Yinsheng. The Mathematic Model of Consciousness In Second Asia International Conference on Modelling and Simulation, 2008.

[2] Sanjay Bhushan. Information Consciousness within the Realm of Creation and its Mathematical Interpretation In International Journal of Research and Innovation in Applied Science (IJRIAS), Volume III, Issue IX, September,ISSN 2454-6194, 2018.

[3] D. M. Armstrong, Norman Malcolm Consciousness and Causality: A Debate on the Nature of Mind In Oxford: Basil Blackwell, 1984.

[4] Hameroff, Stuart and Penrose, Roger Consciousness in the universe: a review of the 'ORCH OR' theory In Physics of life reviews, doi:10.1016/j.plrev.2013.08.002, 2013.

[5] L. S. Penrose, R. Penrose. Impossible Objects: A special type of Visual Illusion In The British Psychological Society journal, https://doi.org/10.1111/j.2044-8295.1958.tb00634.x, 1958.

[6] Giulio Tononi Integrated Information Theory of Consciousness In BMC Neuroscience, 5:42., 2004. 
[7] Tononi, Giulio, and Christof Koch. Consciousness: Here, There and Everywhere? In Philosophical Transactions of the Royal Society, Philosophical Transactions B, 370 (1668).Doi: 10.1098/rstb.2014.0167, 2015.

[8] Roger Penrose. Asymptotic properties of fields and space-times In Phys. Rev. Lett. 10 66-68, 1968.

[9] J A Wheeler. Information, physics, quantum: the search for links In Proceedings III International Symposium on Foundations of Quantum Mechanics, Tokyo, p. 354-368,, 1989.

[10] Wikipedia Pictures on Penrose Space-Time model In https://en.wikipedia.org/wiki/Penrose $e_{d}$ iagram. 